\title{
Role of FNAC in Evaluation of Neck Masses
}

\author{
Mohd Hazmi Mohamed ${ }^{\mathrm{a}, \mathrm{c}}$, Shahrul Hitam ${ }^{\mathrm{b}}$, \\ Sushil Brito-Mutunayagam ${ }^{\mathrm{b}}$, Mohd Razif Mohamad Yunus ${ }^{\mathrm{a}}$
}

\begin{abstract}
Background: Despite fine needle aspiration cytology (FNAC) has a well recognized role in investigation neck masses, insufficient cellular yield in FNA remain an issue. The aim of this study is to evaluate the effectiveness of FNAC as a primary investigation tool in neck masses.
\end{abstract}

Methods: This is a retrospective study of 47 cases with FNAC performed from February 2008 till June 2009 at Otorhinolaryngology Department, Ampang Hospital. The result were compared with results of 44 patients with neck masses and subjected for excision/ incision biopsy as first line investigation of tissue diagnosis during the same period of time.

Results: A total of 37 out of 47 patients with FNAC have a conclusive result (78.7\% diagnostic yield). Inflammatory lesions $(46 \%)$ are the common pathology followed by benign lesions $(41 \%)$ and malignant lesions (13\%). Excision/incision biopsy have 100\% tissue diagnostic yield with $48 \%$ were haematological malignancies followed by reactive lymphadenopathy (27\%), tuberculosis lymphadenopathy (16\%) and metastatic carcinoma (9\%).

Conclusion: Despite lower tissue diagnostic yield compared to excision/incision biopsy, FNAC remains a safe and appropriate first line investigation of neck masses.

Keywords: Fine needle aspiration cytology; Neck masses

Manuscript accepted for publication March 20, 2013

${ }^{a}$ Department of Otorhinolaryngology, University Kebangsaan Malaysia Medical Centre, Kuala Lumpur, Malaysia

${ }^{b}$ Department of Otorhinolaryngology, Ampang Hospital, Malaysia

${ }^{c}$ Corresponding author: Mohd Hazmi Mohamed, Department of Otorhinolaryngology, Medical Faculty, University Kebangsaan Malaysia, Jalan Yaacob Latif, 56000 Cheras, Kuala Lumpur, Malaysia. Email: mdhazmi@outlook.com

doi: http://dx.doi.org/10.4021/jcs178e

\section{Introduction}

Neck masses are a common referral to Otorhinolaryngolgy (ORL) department. Etiologies vary from infective causes to malignant origin. Evaluation of a patient with neck mass should always begin with a thorough history, followed by a complete head and neck examination. If the physical examination does not explain the neck mass, a fine-needle aspiration (FNA) of the mass may be performed. If the mass is believed to be a metastatic lesion, a panendoscopy of the aero-digestive tract is subsequently warranted.

FNAC allows the pathologist to assess the cells aspirated from the lesion. However it does not allow evaluation of the morphology. As a result, false-negative and false-positive results can occur. As FNAC is a simple test, associated with minimal trauma and complication, and results can be rapidly available, it remain a valuable diagnostic tool and is a recommended first line investigations of suspected malignancy cases.

\section{Materials and Method}

This is a retrospective study carried out at Otorhinolaryngology (ORL) Department, Ampang Hospital in which data of patients who had FNAC between February 2008 and June 2009 were identified and reviewed. FNAC procedures were done after a thorough physical examination.

Palpable neck masses were aspirated using $23 \mathrm{G}$ needle and $20 \mathrm{~mL}$ syringe. A negative pressure was applied to the syringe by pulling the syringe plunger and at least two dry specimens and two alcohol fixed specimen were taken. The alcohol fixed smears were immediately submerged in $95 \%$ ethyl alcohol. All the slides preparations were made by ORL department's staff before being sent to department of pathology. Alcohol fixed smears were subsequently stained with Haematoxylin and Eosin (H and E) stains.

The data were divided into four large groups which represent the nature of origin of the neck masses. The groups are: a) salivary glands, b) cervical lymph nodes, c) thyroid gland, and d) undetermined origin. The results of the FNAC 
Table 1. The Results of First and Second Attempt of FNAC

\begin{tabular}{llll}
\hline & $\begin{array}{l}\text { Satisfactory and } \\
\text { conclusive }\end{array}$ & Unsatisfactory & Inconclusive \\
\hline First attempt $(\mathrm{n}=47)$ & $31(66 \%)$ & $6(13 \%)$ & $10(21 \%)$ \\
Second attempt $(\mathrm{n}=6)$ & $6(13 \%)$ & $0(0)$ & $0(0)$ \\
\hline
\end{tabular}

were evaluated into five categories which are: 1) unsatisfactory sample, 2) inconclusive, 3) inflammatory process, 4) benign disorder, and 5) malignant disorder.

Open biopsy of head and neck masses was carried out when FNAC result was unsatisfactory after two attempts or if FNAC result suggested for biopsy as further investigation.
Comparisons were made with patients who presented with neck masses and were subjected for excision or incision biopsies during the same period (February 2008 - June 2009) as a first line investigation. A total of 44 cases fulfilled the criteria, 20 cases were known to have haematological malignancy and in these cases, excision biopsy was done to

Table 2. Results of FNAC of Neck Swelling, Showing the Relative Frequencies of Various Pathological Conditions $(\mathrm{n}=37)$

\begin{tabular}{|c|c|c|}
\hline Diagnosis & No. of cases/ $(\%)$ & Action \\
\hline \multicolumn{3}{|l|}{ Lymphadenitis } \\
\hline Reactive & $8(21 \%)$ & Conservative management \\
\hline Tuberculous & $4(11 \%)$ & Referral to respiratory team \\
\hline Sialadenitis & $5(14 \%)$ & Conservative management \\
\hline \multicolumn{3}{|l|}{ Benign lesions } \\
\hline Salivary Gland & $6(16 \%)$ & \\
\hline Pleomorphic adenoma & -4 & -Surgical resection (HPE and FNAC corresponded) \\
\hline Benign mass & -2 & -Conservative management \\
\hline Others & $4(11 \%)$ & Surgical excision (HPE and FNAC corresponded) \\
\hline Cystic lesions & -4 & \\
\hline \multicolumn{3}{|l|}{ Malignant lesions } \\
\hline Lymph node & $3(8 \%)$ & \\
\hline Thyroid papillary carcinoma & -2 & Referral to surgical department \\
\hline Nasopharyngeal carcinoma & -1 & Referred to Oncologist \\
\hline Others & $2(5 \%)$ & \\
\hline Carcinoma of unknown primary & -1 & Radical neck dissection and adjuvant radiotherapy \\
\hline Rhabdomyosarcoma & -1 & Referred to oncologist \\
\hline \multicolumn{3}{|l|}{ Thyroid } \\
\hline Benign & $5(14 \%)$ & \\
\hline Goitre & -4 & Referral to surgical department \\
\hline Thyroglossal cyst & -1 & $\begin{array}{l}\text { Conservative management since it's the only function } \\
\text { thyroid on thyroid scan }\end{array}$ \\
\hline
\end{tabular}


Table 3. HPE Results of Patients With Inconclusive or Unsatisfactory Sample of FNAC

\begin{tabular}{ll}
\hline FNAC report & HPE results \\
\hline Inconclusive & \\
a) cervical lymph node & \\
1. suspicious cell seen & Hodgkin Lymphoma $(\mathrm{n}=3)$ \\
2. no atypical cell seen & TB lymphadenitis $(\mathrm{n}=1)$ \\
b) salivary gland & Sialedinitis $(\mathrm{n}=1)$ \\
3. atypical cell seen & \\
a) cervical lymph node & \\
4. unsatisfactory sample & TB lymphadenitis $(\mathrm{n}=1)$ \\
b) undetermined & \\
5. unsatisfactory sample & \\
6. unsatisfactory sample & Intramuscular lipoma $(\mathrm{n}=1)$
\end{tabular}

exclude recurrence of haematological malignancy.

\section{Results}

A total of 47 cases fulfilled the criteria. The age of the patients ranged from 13 to 72 years with the mean age of 50 - 60 years old. There were 25 male and 22 female patients. Most of the patients are Malay $(\mathrm{n}=31)$ followed by Chinese $(\mathrm{n}=13)$, Indian $(\mathrm{n}=2)$ and others $(\mathrm{n}=1)$.

Majority of aspirates were from cervical lymph node which consists of 20 cases $(43 \%)$ followed by salivary gland with 12 cases $(26 \%)$, undetermined origin with 10 cases $(21 \%)$ and thyroid gland with 5 cases $(10 \%)$.

On first attempt of FNAC, 31 samples were satisfactory and have conclusive results $(66 \%)$, shown in Table 1. Out of 16 cases which reported as unsatisfactory or inconclusive, FNAC were re-attempted for second time in 6 cases and all of them gained a satisfactory result. Based on the accumulative data of both first attempt and repeated FNAC, 37 out of 47 cases have satisfactory results $(78.7 \%)$.

Reactive lymphadenitis was the commonest diagnosis (21\%) followed by salivary benign lesion mostly pleomorphic adenoma. Other pathologies include sialedinitis (14\%), malignant neoplasm (13\%) and tuberculous lymphadenitis $(11 \%)$. Metastatic carcinoma to lymph nodes was the most common case of malignancy. The results are tabled in Table 2.

Of 10 patients which FNAC were group as inconclusive or unsatisfactory sample, 8 of them were subjected for open biopsy. Two patients whose initial FNAC were inconclusive declined further investigations that included open biopsy. The results are tabled in Table 3.

During the same period, 44 patients had excision or incision biopsy done as the first line investigations with 20 of them with history of haematological malignancies. Among the 20 patients previously treated for underlying haematological malignancies, 19 were positive for relapse and one case had infective lymphadenitis.

Among the other 24 cases which presented with new findings of palpable cervical lymph nodes; 11 were reported as reactive lymph node, 7 were reported as TB lymphadenitis, 2 were suspected to have haematological malignancy that were later confirmed as B lymphocytic leukemia and non-Hodgkin lymphoma respectively and 4 cases with nonhaematological malignancies. Among these 4 patients; 2 cases had Nasopharyngeal Carcinoma (NPC), 1 case with metastatic carcinoma of prostate and 1 case with metastatic carcinoma of unknown primary (Fig. 1).

\section{Discussion}

Our review showed a conclusive diagnostic yield of 78.7\% for FNAC in evaluation of neck masses in our centre. This conclusive diagnostic yield includes repeated FNACs after failure of the first attempt FNAC. Our unsatisfactory sample on first attempt of FNAC was 6 out 47 patients (13\%) and still below acceptable range unsatisfactory sample as suggested by guidelines of the Papanicolaou Society of Cytopa- 


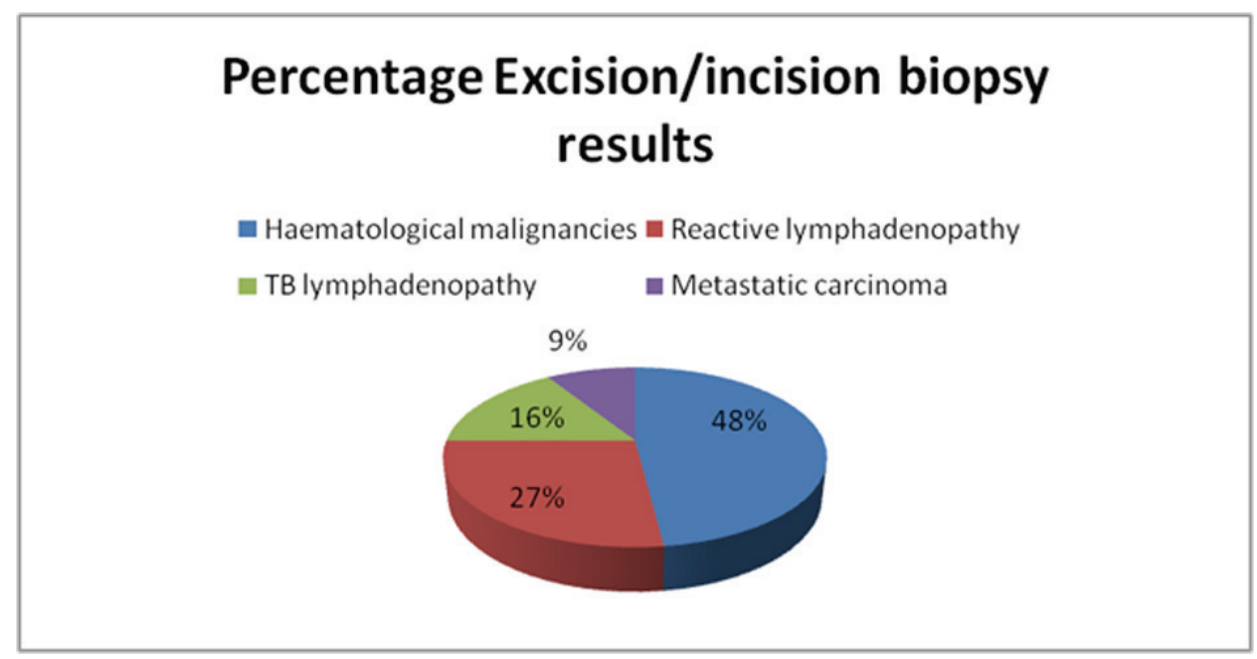

Figure 1. Percentage excision/incision biopsy results based on etiology.

thology for fine-needle aspiration procedure and reporting; which is below $15 \%$ [1].

In our study, inflammatory lesions which consist of lymphadenitis and sialedinitis were the most common pathology accounting for $46 \%$. This is followed by benign neoplasm (41\%) and malignant neoplasm accounted for $13 \%$. Most of the inflammatory lesions were due to reactive lymphadenits (21\%). El-Hag et al showed that reactive/non-specific lymphadenitis was the most common (33\%) cause of neck masses among 225 Saudi patients [2]. Tuberculous lymphadenitis was the next most common pathology (21\%) followed by malignant neoplasms (13\%). Similarly, a study in India found that $84.5 \%$ cases to be benign lesions and $15.5 \%$ cases of malignant lesions in the evaluation of asymptomatic cervical lymphadenopathy [3]. In contrast, the spectrum of findings is different in the more developed nations. A study from New Zealand $(n=187)$ showed that malignancy accounted for $50 \%$ of cases [4]. Similarly, another study from the west reported $59 \%$ to be malignant lesions and $34 \%$ to be benign lesions [5]. This shows epidemiological variation between the developed and developing countries. Infections like TB are common in developing countries while malignancies are more common in developed countries. Malaysia is a developing country and the spectrum shows a similar pattern to other developing countries where cervical lymphadenitis to be more common cause of neck masses.

Use of FNAC for evaluating isolated salivary gland masses is generally accepted. However, the results of FNAC should be interpreted taking into account the clinical and investigative findings. Costas et al reported sensitivity and specificity of $84.8 \%$ and $93.7 \%$, respectively for FNA of salivary gland $(n=112)[6]$. Cohen et al reported a satisfactory specimen was obtained in $89 \%$ among 169 patients and compared to histological diagnoses, the positive and negative predictive values of FNA for salivary glands were $84 \%$ and
$77 \%$, respectively [7]. These results suggested that FNAC alone may not be sufficient to rule out salivary malignancies and that further clinical evaluation, including surgical resection, may be necessary. In our study, all four patient diagnoses of pleomophic adenoma on FNAC were confirmed after surgical resection. Unfortunately, two patients who were diagnosed with benign lesions did not proceed to surgery.

Despite being inferior to excision biopsy, there are advantages of FNAC. First, the sensitivity and specificity of FNAC is sufficiently high and is considered acceptable. Second, FNAC is cost effective. In the era of economic recession, cost savings is an important consideration. The cost of excision biopsy is much higher especially if a hospital stay is required and time off from work is needed. Furthermore, preoperative evaluations that include blood, radiological and occasional cardiac investigations generally required prior to general anaesthesia will incur additional cost. Third, FNAC also is safer; obviate the risks of anaesthesia, tumour seeding, postoperative complications and scarring. Cosmetic is certainly a very important issue to be considered when dealing with masses in the area of head and neck especially in female patients. Furthermore, results of FNAC are available faster than biopsy.

Generally, FNAC is a safer choice for the initial evaluation masses suspicious of malignancy as the frequency of needle seeding of the tumor cells in FNAC procedure is reported as low as $0.003-0.009 \%$ [8]. Hence the risk of tumour seeding in FNAC is almost nonexistent in contrast to excision biopsy which has significant risk of tumour seeding causing distant metastases of cancer itself.

The main limitation with our study was the small sample size. Second the department was newly established and hence the findings may not be comparable to established centres. However, despite this our yield can be considered acceptable and given time, this yield is likely to increase 
once the department becomes more established.

\section{Conclusion}

In conclusion, our study shows that FNAC remains an appropriate first line investigation of head and neck masses. FNAC is an economical, fast, and minimally invasive with acceptable sensitivity and few complications compared to open biopsy.

\section{Grant Support}

Nil.

\section{References}

1. Guidelines of the Papanicolaou Society of Cytopathology for fine-needle aspiration procedure and reporting. The Papanicolaou Society of Cytopathology Task Force on Standards of Practice. Diagn Cytopathol. 1997;17(4):239-247.

2. el Hag IA, Chiedozi LC, al Reyees FA, Kollur SM. Fine needle aspiration cytology of head and neck masses. Seven years' experience in a secondary care hospital. Acta Cytol. 2003;47(3):387-392.

3. Sarda AK, Bal S, Singh MK, Kapur MM. Fine needle aspiration cytology as a preliminary diagnostic procedure for asymptomatic cervical lymphadenopathy. J Assoc Physicians India. 1990;38(3):203-205.

4. Cheng AT, Dorman B. Fine needle aspiration cytology: the Auckland experience. Aust N Z J Surg. 1992;62(5):368-372.

5. Steel BL, Schwartz MR, Ramzy I. Fine needle aspiration biopsy in the diagnosis of lymphadenopathy in 1,103 patients. Role, limitations and analysis of diagnostic pitfalls. Acta Cytol. 1995;39(1):76-81.

6. Costas A, Castro P, Martin-Granizo R, Monje F, Marron C, Amigo A. Fine needle aspiration biopsy (FNAB) for lesions of the salivary glands. Br J Oral Maxillofac Surg. 2000;38(5):539-542.

7. Cohen MB, Ljung BM, Boles R. Salivary gland tumors. Fine-needle aspiration vs frozen-section diagnosis. Arch Otolaryngol Head Neck Surg. 1986;112(8):867-869.

8. DeMay RM.The art and science of cytopathology. Volume II:aspiration cytology. Chicago: ASCP Press.1996;464-74. 